\title{
Impacto de intervenção educativa sobre feridas no conhecimento de técnicos de enfermagem
}

\author{
Educational intervention about wounds: impact on nursing technicians' knowledge \\ Impacto de intervención educativa sobre heridas en el conocimiento de los técnicos en enfermería
}

\author{
Oleci Pereira Frota ${ }^{I}$; Jaqueline Goulart de Oliveira Constanci ${ }^{I I}$; Marisa Dias Rolan Loureiro ${ }^{I I I}$; Adriano Menis Ferreira ${ }^{I V}$
}

\begin{abstract}
RESUMO: Objetivou-se avaliar o efeito de uma intervenção educativa no conhecimento da equipe técnica de enfermagem sobre aspectos do cuidado com feridas. Estudo descritivo-exploratório comparativo, desenvolvido de agosto a novembro de 2012 em 12 unidades de atenção primária à saúde do interior do Estado de Mato Grosso do Sul, Brasil. Os dados foram coletados de 23 profissionais antes e após intervenção educativa, utilizando um teste de conhecimento. De maneira geral, a intervenção proporcionou melhora no nível de conhecimento dos profissionais $(p=0,001)$. Entretanto, somente os tópicos limpeza, tecido necrótico, avaliação da evolução e pomada sulfadiazina de prata foram considerados conhecidos por $\geq 90 \%$ dos participantes. Em três tópicos, não houve aumento na proporção de acertos pós-teste: desbridamento autolítico ( $p=0,179)$, tecido com granulação $(p=0,067)$ e avaliação da evolução da ferida $(p=0,108)$. Conclui-se que a intervenção educativa não foi totalmente eficaz. Faz-se necessário buscar estratégias coadjuvantes, a fim de proporcionar conhecimento desejável à amostra estudada.
\end{abstract}

Palavras-Chave: Educação em enfermagem; enfermagem baseada em evidências/educação; capacitação em serviço; curativos.

ABSTRACT: This exploratory, comparative, descriptive study to evaluate the effect of an educational intervention on the nursing technicians' knowledge on aspects of wound care was conducted at 12 primary health care facilities in Mato Grosso do Sul State, Brazil, from August to November 2012. Using a test of knowledge, data were collected from 23 subjects before and after the educational activity. Overall, the activity improved the professionals' levels of knowledge $(p=0.001)$. However, only the topics 'cleansing', 'necrotic tissue', 'assessing progress', and 'silver sulfadiazine ointment' were considered known by $\geq 90 \%$ of participants. There was no increase in the proportion of correct post-test answers on three topics: 'autolytic debridement' ( $p=0.179)$, 'granulation tissue' ( $p=0.067)$ and 'assessment of wound progress' $(p=0.108)$. It was concluded that the educational intervention was not fully effective. Supporting strategies should be sought to provide the desired knowledge to the sample studied.

Keywords: Nursing education; evidence-based nursing/education; in-service training; dressings.

RESUMEN: Este estudio tuvo como objetivo evaluar el efecto de una intervención educativa en el conocimiento del equipo de técnicos de enfermería sobre los aspectos del cuidado de heridas. Estudio exploratorio descriptivo comparativo, realizado entre agosto y noviembre de 2012, en 12 unidades de atención primaria de salud en el interior del estado de Mato Grosso do Sul, Brasil. Los datos se obtuvieron de 23 profesionales antes y después de la intervención educativa, mediante una prueba de conocimientos. En general, la intervención proporcionó una mejoría en el nivel de conocimiento de los profesionales ( $\mathrm{p}=0,001$ ). Sin embargo, sólo los temas limpieza, tejido necrótico, evaluación de la evolución de la herida y la crema sulfadiazina de plata se consideraron conocidos por el $\geq 90 \%$ de los participantes. En tres rubros no hubo un aumento en la proporción de éxitos después de la prueba: desbridamiento autolítico $(p=0,179)$, tejido de granulación $(p=0,067)$ y evaluación de la evolución de la herida $(p=0,108)$. Se concluye que la intervención educativa no fue plenamente eficaz. Es necesario buscar estrategias de apoyo para proporcionar conocimiento deseable a la muestra estudiada.

Palabras Clave: Educación en enfermería; enfermería basada en evidencia/educación; capacitación en servicio; curativos.

\section{INTRODUÇÃO}

A educação é um processo contínuo que en globa processos de ensino-aprendizagem na busca da construção do conhecimento, em que se utiliza o pensamento livre, crítico e reflexivo. Contribui para justificar um compromisso pessoal e profissional, a fim de capacitar e transformar a realidade vivenciada. A educação em saúde, especificamente, representa ativi- dades desenvolvidas por meio de programas educativos, com o objetivo de orientar os profissionais, seguindo os princípios institucionais. É desenvolvida para oportunizar ao profissional a ampliação da qualidade de suas capacidades e competências, buscando cumprir suas responsabilidades, e, ainda, garantir uma assistência com menor probabilidade de eventos adversos ${ }^{1}$.

'Enfermeiro. Mestre em Saúde e Desenvolvimento na Região Centro-Oeste. Doutorando pelo Programa de Pós-Graduação em Saúde e Desenvolvimento na Região Centro-Oeste da Universidade Federal de Mato Grosso do Sul. Campo Grande, Mato Grosso do Sul, Brasil. E-mail: olecifrota@gmail.com. IIEnfermeira. Especialista em Estomaterapia. Prefeitura Municipal de Três Lagoas. Três Lagoas, Mato Grosso do Sul, Brasil. E-mail: jaque gou@yahoo.com.br IIIEnfermeira. Professora Adjunta e Coordenadora do Programa de Mestrado em Enfermagem da Universidade Federal de Mato Grosso do Sul. Campo Grande, Mato Grosso do Sul, Brasil. E-mail: marisarolan@gmail.com.

IV Enfermeiro. Professor Adjunto do Curso de Enfermagem e Orientador do Programa de Mestrado e Doutorado em Saúde e Desenvolvimento na Região Centro- Oeste e Mestrado em Enfermagem da Universidade Federal de Mato Grosso do Sul. Campo Grande, Mato Grosso do Sul, Brasil. E-mail: a.amr@ig.com.br. 
Destaca-se que a gestão de pessoas, no campo da saúde, está voltada à capacitação profissional para o desenvolvimento de recursos humanos $(\mathrm{RH})$ com habilidades cognitivas e tecnológicas, com foco na educação reflexiva e participativa, em oposição ao treinamento exclusivamente técnico-operacional, o qual visa à ampliação de capacidades psicomotoras. Nessa perspectiva, estudar o processo de aprendizagem no ambiente organizacional pode gerar novas descobertas e contribuir para mudanças na prática, na gestão e na administração².

Desde seu surgimento como profissão, a enfermagem sempre esteve inserida no papel de principal cuidador de pessoas com feridas. Com os avanços tecnológicos nesta área, obtiveram-se novos métodos para avaliar, monitorar e diagnosticar feridas e pacientes; concomitantemente, surgiram produtos e terapias inovadoras. Isso implica a necessidade de se buscar qualificações, atualizações, habilidades psicomotoras e aptidões cognitivo-tecnológicas, bem como conhecimento técnico-científico para o adequado atendimento à pessoa com feridas ${ }^{3,4}$.

Entretanto, atualmente, observa-se um paradoxo entre os conhecimentos requeridos para cuidar de pessoas acometidas por feridas e o que está sendo ensinado nos cursos Técnicos e de Graduação em Enfermagem, haja vista que o conhecimento oferecido - particularmente pelos cursos de graduação - é insuficiente ${ }^{5}$. Além disso, atualmente, parece não haver consenso sobre qual disciplina pedagógica deva ser responsável pelos ensinamentos do cuidado de pacientes com feridas. Como conhecimento deficiente leva a práticas inadequadas, infere-se que as práxis atuais são influenciadas pela cultura local e ritualismo, em oposição às recomendações das diretrizes baseadas em evidências ${ }^{4}$.

Considerando os benefícios que a educação em saúde pode proporcionar à melhoria da qualidade da assistência, gerência e administração em enfermagem, questiona-se: Qual é o efeito provocado pela implantação de uma intervenção educativa sobre pacientes com feridas, utilizando, como estratégia, um curso, no nível de conhecimento dos profissionais de enfermagem da atenção primária à saúde? Para responder a essa questão, propõe-se a realização deste estudo, que tem por objetivo verificar o efeito de uma intervenção educativa no conhecimento dos técnicos de enfermagem sobre avaliação, manejo e tratamento tópico de feridas.

Justifica-se a realização deste estudo porque, em busca realizada nas principais bases eletrônicas de dados, não foram encontradas pesquisas que retratassem o efeito de intervenções educativas no conhecimento da equipe de enfermagem de atenção primária sobre feridas. As bases pesquisadas foram a de Literatura Latino-Americana e do Caribe em Ciências da Saúde (LILACS), Banco de Dados em Enfermagem (BDENF), Medical Literature Analysis and Retrieval System on-line (MEDLINE) e Scientific
Electronic Library Online (SCIELO), com o cruzamento dos descritores controlados (Descritores em Ciências da Saúde-DeCS) Training x Nursing Team x Wound Healing e Learning x Nursing Team x Wound Healing. Nessa busca, foram identificados, apenas, estudos semelhantes, porém em nível hospitalar e abordando, exclusivamente, úlceras por pressão ${ }^{6-8}$.

\section{REVISÃo DE Literatura}

Na área da saúde, o processo de trabalho vem sendo substancialmente alterado pelas notáveis mudanças sanitárias ocorridas nas últimas décadas, políticas de saúde e demandas da população, além das modificações contextuais advindas do progresso científico e tecnológico no cenário mundial. Forçadas a atuarem em consonância com as novas exigências - ou mesmo a antecipá-las -, as organizações, em busca de vantagens competitivas que permitam diferenciá-las de seus concorrentes, conferem à área de $\mathrm{RH}$ um posicionamento estratégico. Em contrapartida, passam a exigir demonstrativos concretos da efetividade de suas ações. Nesse contexto, o principal desafio, atualmente imposto à área de RH, é tornar prioritária a administração do capital humano, igualando-a a qualquer outro recurso essencial para o desenvolvimento institucional ${ }^{9}$.

A implantação de ações educativas organizadas, sistematizadas e voltadas à qualificação contínua de profissionais de saúde constitui-se uma estratégia de fundamental importância para assegurar e incrementar a qualidade da atenção à saúde. Dentre elas, destaca-se, indiscutivelmente, a enfermagem, tema de debate no contexto atual da política pública de saúde brasileira. As análises crítico-reflexivas e os consensos construídos com base nessas discussões resultaram na implantação da Política Nacional de Educação Permanente em Saúde ${ }^{10,11}$. Essa política recomenda que o profissional de saúde seja formado e que suas capacidades sejam permanentemente ampliadas segundo as necessidades de saúde regional e dos processos de mudança do modelo assistencial e gerencial na perspectiva da integralidade, da prática multi e interprofissional e da efetividade da atenção à saúde ${ }^{12}$.

O treinamento e desenvolvimento de RH podem ser entendidos como três subsistemas coordenados: a avaliação de necessidades, o planejamento e execução e a avaliação do treinamento, sendo que o último fornece informações sistematizadas sobre o conjunto do treinamento, enquanto os demais se referem ao desenvolvimento do treinamento ${ }^{2}$. Assim, antes da implementação de qualquer intervenção educativa, faz-se precípuo o diagnóstico de necessidades de treinamento que envolve a aplicação de meios, métodos e técnicas que visam à identificação de lacunas das competências exigidas para um desempenho humano adequado no trabalho ${ }^{13}$. 
Diante dessa realidade, as organizações vêm investindo muito em educação continuada e permanente: comissões e setores já estão presentes em várias instituições de saúde do Brasil. Entretanto, observa-se a necessidade de uma atuação mais efetiva e fundamentada em princípios científicos. Na maioria dos casos, as ações educativas são feitas às cegas, ou seja, as atividades são oferecidas sem o devido estudo criterioso prévio das reais necessidades da população-alvo, podendo resultar em dispêndios de tempo e recursos financeiros. Além disso, muitos treinamentos, cursos e capacitações são oferecidos sem uma avaliação de sua eficácia e eficiência em termos de impacto no trabalho ${ }^{14}$.

Muito se discute sobre a aplicação de cuidados avançados em saúde, entre os quais a preocupação com a avaliação, o manejo e o tratamento das feridas. Mas, para tanto, faz-se necessário envolver os profissionais de enfermagem com saberes e fazeres específicos, com a finalidade de proporcionar cuidado individualizado, humanizado e holístico, buscando compreender o contexto fisiopatológico em que o paciente se encontra e a terapêutica adequada, sem deixar de se preocupar, no entanto, com os fatores psicológicos, sociais e ambientais particulares de cada indivíduo ${ }^{15}$.

A relevância de possuir aptidão e buscar continuamente conhecimentos científicos para prestar cuidados de excelência a pacientes com feridas é destacada por vários autores ${ }^{4,6-8,15}$, dada a constatação que esta é uma área na qual, frequentemente, a prática é baseada em mitos, tradições e senso comum. Há de se considerar que a rápida evolução de terapias tópicas e coberturas mudaram as práticas de assistência aos pacientes com feridas e deu origem a novas recomendações profissionais ${ }^{4}$. Essas e outras condições impõem a necessidade de se avaliar os conhecimentos e as práticas dos profissionais de enfermagem em diversos cenários, identificando se há a necessidade de intervenções educativas, bem como sua implementação.

\section{Metodologia}

Trata-se de estudo quantitativo com delineamento descritivo-exploratório comparativo, desenvolvido de agosto a novembro de 2012, em oito unidades de saúde das estratégias de saúde da família (ESF) e cinco estratégias de agentes comunitários de saúde (EACS) do interior do Estado de Mato Grosso do Sul, Brasil. O estudo foi aprovado pelo Comitê de Ética em Pesquisa com Seres Humanos sob o Protocolo $n^{\circ} 47780 / 2012$.

Uma vez que o curso foi específico para a equipe técnica de enfermagem, todos os profissionais lotados nos setores investigados foram convidados a participar e foi obtido o Termo de Consentimento Livre e Informado. Foram excluídos aqueles que estiveram de licença ou férias durante o período da coleta de dados, que tinham tempo de atuação na área inferior a um ano e os que não participaram de todas as atividades educativas propostas ou não responderam integralmente o pré e pós-teste. A população foi representada por 40 profissionais que compunham o quadro da equipe técnica de enfermagem no período de estudo. Após aplicação dos critérios de seleção, a amostra foi constituída por 23 sujeitos.

Para atender ao objetivo desta investigação, foi construído um questionário estruturado composto por itens referentes aos dados sociodemográficos (variável independente) e teste de conhecimento (variável dependente). Este último visou identificar o nível de conhecimento dos participantes sobre temas relacionados à avaliação e tratamento de feridas, antes e após a intervenção educativa. $\bigcirc$ referido instrumento foi elaborado com base na literatura vigente, nas experiências dos pesquisadores e, ainda, considerando os recursos terapêuticos disponíveis e as práticas realizadas nas referidas unidades pelos profissionais aos pacientes com feridas.

Ressalta-se que foram tomados os devidos cuidados quanto à elaboração das questões que compuseram o instrumento de avaliação do conhecimento, haja vista a preocupação de não elencar conhecimentos e práticas inerentes ao exercício privativo do enfermeiro ou outros profissionais de saúde.

Com a finalidade de verificar a objetividade, pertinência e clareza dos itens, o instrumento de coleta de dados foi submetido à validação de conteúdo por três juízes com conhecimento técnico-científico acerca do assunto (dois pesquisadores e uma enfermeira assistencial da atenção primária). Após refinamentos sugeridos, foi realizado um teste-piloto com a aplicação do questionário em 10 profissionais da equipe técnica de enfermagem lotados em uma ESF de outro município, para avaliar sua operacionalidade, objetividade e compreensão semântica.

Após apontamentos e adequações, a versão final, quanto ao componente teste de conhecimento, foi constituída por 13 tópicos contendo de cinco a 12 afirmações cada. Foi solicitado aos sujeitos que respondessem ao teste, tendo como opções para cada item, verdadeiro $(\mathrm{V})$, quando concordassem com a afirmação, e falso $(\mathrm{F})$, quando discordassem. Foram considerados acertos às questões $\mathrm{V}$ respondidas como verdadeiras ou F respondidas como falsas. Para que um tópico fosse considerado como conhecido pelo profissional, $90 \%$ de suas questões deveriam ser respondidas corretamente $^{8,16}$.

estudo foi dividido em três momentos: aplicação do questionário fechado (pré-teste: visando determinar se havia a necessidade da atividade educativa), educação em serviço e, por último, a reaplicação do questionário fechado (pós-teste). Conforme os resultados do pré-teste, a intervenção educativa foi 
indicada e todos os participantes foram convidados, direta e indiretamente (panfletos), a participarem de um curso com carga horária de 40 horas intitulada Atualização de enfermagem no cuidado a pacientes com feridas. Foi realizada breve explanação sobre objetivos do curso, funcionamento, carga horária, conteúdo programático, dentre outros, assim como a realização deste estudo e a importância da participação dos profissionais na qualidade de voluntários.

Organizou-se ação educativa, na forma de oficinas de cunho teórico-prático, desenvolvida em um encontro semanal, utilizando diferentes estratégias como aula expositiva dialogada, discussão em grupo sobre a prática vigente (considerando a realidade local), estudos de casos, e fornecimento aos sujeitos de um guia impresso (conteúdo e questões de fixação). Um cronograma de execução foi elaborado e fornecido aos participantes. Destaca-se que os conteúdos abordados foram baseados na realidade e disponibilidade de insumos e equipamentos locais. Cada encontro teve duração de aproximadamente 60 minutos e foram conduzidos pela enfermeira-pesquisadora.

O primeiro e o último encontro (primeiro e terceiro momento) foram destinados à aplicação do pré e pós-teste, respectivamente. Cabe destacar que a aplicação do pós-teste foi executada 15 dias após ação educativa. Os demais encontros foram designados para a ação em saúde propriamente dita (segundo momento), distribuída da seguinte forma: conceito de ferida; tipos de reparação tecidual; tipos de cicatrização; fatores que interferem na cicatrização; avaliação sumária do portador de ferida; úlceras crônicas: pé diabético, úlcera por pressão e venosa; conceitos de infecção e colonização; acrômio: Red, Yellow, Black (RYB); limpeza, desbridamento e mensuração e; coberturas/ tratamentos: vaselina, neomicina, sulfadiazina de prata, papaína, colagenase, ácidos graxos essenciais (AGE), alginato de cálcio, hidrocolóide, hidrogel, carvão ativado com prata.

Os dados foram dispostos em um banco de dados utilizando-se o software Microsoft Office Excel versão 2010. Considerou-se conhecimento suficiente, para cada tópico e média global, quando $\geq 90 \%$ dos profissionais responderam corretamente tanto no pré quanto no pós-teste. A análise estatística considerou os acertos pré e pós-teste e não os acertos isolados para cada sujeito. Visando identificar a existência de diferença estatisticamente significante entre os dois momentos (pré e pós-intervenção educativa), foi aplicado o teste de Wilcoxon com nível de significância de $5 \%(\mathrm{p}<0,05)$.

\section{Resultados e Discussão}

Participaram do estudo 23 profissionais de nível técnico da equipe de enfermagem, sendo todos do sexo feminino. A média da idade foi de 38,04 anos \pm 9,68. Quanto ao tempo de formação profissional, a média alcançou 7,52 anos $\pm 5,35$ e para o tempo de experiência na função, a média atingiu 8,69 anos $\pm 5,01$.

Os resultados referentes ao pré e pós-teste sobre avaliação, manejo e tratamento tópico de feridas são apresentados na Tabela 1.

Os dados percentuais da frequência de acertos de cada um dos 13 tópicos foram transformados em notas de zero a 10 - isto é, o percentual de acerto dos tópicos foi dividido por 10 - e construída a Figura 1. Esta ilustra os valores estatísticos descritivos e de dispersão referentes às notas dos participantes segundos os momentos pré e pós-treinamento.

TABELA 1: Índices de acertos dos profissionais nas questões relativas aos cuidados com portadores de feridas, nos testes pré e pós-intervenção educativa. Três Lagoas/MS, 2012. (N=23)

\begin{tabular}{|c|c|c|c|c|c|}
\hline \multirow{3}{*}{ Questões relativas aos tópicos } & \multicolumn{4}{|c|}{ Frequência de acertos } & \multirow{3}{*}{$\mathbf{p}^{(*)}$} \\
\hline & \multicolumn{2}{|c|}{ Pré-teste } & \multicolumn{2}{|c|}{ Pós-teste } & \\
\hline & f & $\%$ & $f$ & $\%$ & \\
\hline 1- Limpeza & 15 & 65,2 & 22 & 96,7 & 0,018 \\
\hline 2-Tecido necrótico & 13 & 56,5 & 22 & 96,7 & 0,007 \\
\hline 3- Desbridamento autolítico & 13 & 56,5 & 15 & 65,2 & 0,179 \\
\hline 4- Ferida com cavidade & 7 & 30,4 & 13 & 56,5 & 0,027 \\
\hline 5-Tecido com granulação & 13 & 56,5 & 17 & 73,9 & 0,067 \\
\hline 6- Avaliação da evolução da ferida & 20 & 87,0 & 23 & 100 & 0,108 \\
\hline 7- Resposta ao tratamento instituído & 10 & 43,5 & 18 & 78,3 & 0,011 \\
\hline 8- Processo de cicatrização & 13 & 56,5 & 20 & 87,0 & 0,018 \\
\hline 9- Pomada Colagenase & 12 & 52,2 & 18 & 78,3 & 0,027 \\
\hline 10- Pomada Sulfadiazina de Prata & 15 & 65,2 & 21 & 91,3 & 0,027 \\
\hline 11- Pomada Neomicina & 9 & 39,1 & 18 & 78,3 & 0,007 \\
\hline 12-Petrolato ou Vaselina & 12 & 52,2 & 19 & 82,6 & 0,018 \\
\hline 13-Óleo com AGE & 12 & 52,2 & 19 & 82,6 & 0,018 \\
\hline Média global & 12,61 & 54,84 & 15,46 & 82,1 & 0,001 \\
\hline
\end{tabular}

(*)Teste de Wilcoxon 


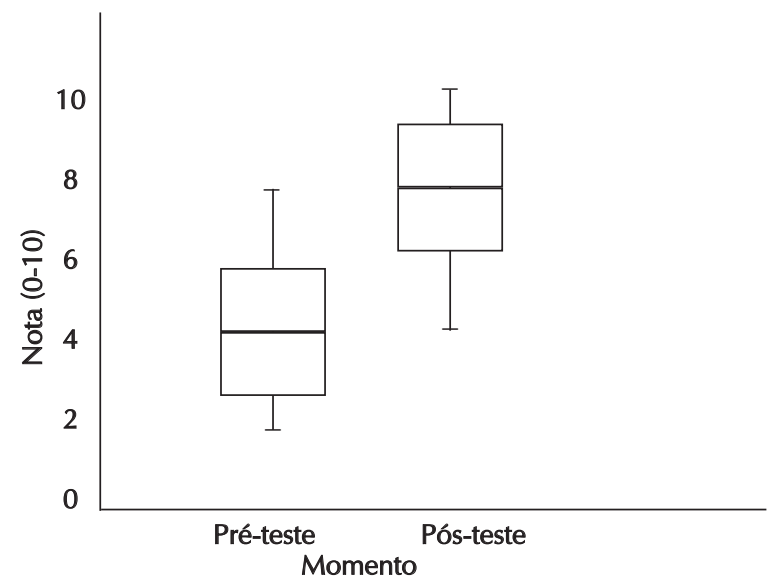

FIGURA 1: Box-plot das notas dos participantes, segundo os pré e pós-teste. Três Lagoas/MS, 2012.

Os resultados obtidos revelam que a intervenção educativa proporcionou efeito positivo na média global de acertos do questionário sobre cuidados com portadores de feridas. Na grande maioria dos tópicos, houve aumento estatisticamente significante na proporção de acertos globais nas respostas do questionário no pós-teste. Assim, pode-se afirmar que profissionais tendem a responder corretamente as questões após intervenção educativa. Esses achados sugerem que os métodos empregados foram favoráveis à aprendizagem, constatado, também, pelo interesse e participação dos profissionais nas atividades propostas.

Apesar disso, não foi adequado levar em conta apenas p-valor para assegurar que essa intervenção é capaz de garantir que os participantes detêm conhecimento teórico suficiente para cuidar de pacientes com feridas, tampouco práticas. Pela análise da Tabela 1, percebe-se que, em apenas quatro tópicos $(1,2,6$ e 10), mais de $90 \%$ dos profissionais acertaram, pelo menos, 90\% das questões após a intervenção. Tendo em vista o referencial teórico adotado ${ }^{7,8}$, constatou-se que o conhecimento adquirido após intervenção foi insuficiente para um profissional que recém concluiu um curso sobre o assunto. Por outro lado, não se pode deixar de considerar a subjetividade de atribuir uma nota de corte para classificar conhecimento adequado ou não sobre um assunto de interesse. Ainda, há de se considerar que o conteúdo possa ter sido demasiadamente complexo.

A capacidade de programas educacionais, cursos de certificação, participação em atualizações e busca na internet em alcançar o conhecimento sobre feridas foi confirmada em estudo que incluiu 460 enfermeiros americanos com diferentes níveis educacionais. Nesse estudo, os enfermeiros que obtiveram certificação para o cuidado com feridas atingiram $89 \%$ de acertos no teste de conhecimento sobre úlcera por pressão, contra $76,5 \%$ daquelas que não tinham certificação ${ }^{8}$.
As questões relativas aos tópicos 4, 7 e 11, mostradas na Tabela 1 , sobressaem entre as que apresentaram maior porcentagem de erros no questionário pré-teste, denotando frágil conhecimento prévio acerca dos cuidados com ferida cavitária, avaliação da resposta ao tratamento instituído e uso de pomada neomicina. Além disso, observa-se que os mesmos tópicos permanecem com maior percentual de erros após intervenção educativa, demonstrando maior dificuldade dos participantes em aprender ou aceitar/ compreender a importância de aspectos relacionados às temáticas. Estes achados indicam que os referidos tópicos devem ser melhor trabalhados durante as atividades educativas. Além disso, recomenda-se que estudos de natureza qualitativa sejam realizados para melhor compreender esse fenômeno.

Geralmente, as questões dos testes que se referem a conteúdos novos ou desconhecidos são essenciais para avaliar o efeito de uma intervenção educativa na aquisição de conhecimento, pois se espera menor índice de acertos no pré e maior no pós-teste ${ }^{2}$. Embora perceba-se aumento do nível de conhecimento no pós-teste nas questões concernentes aos tópicos 4, 7 e 11 (respectivamente, ferida com cavidade, resposta ao tratamento instituído e pomada neomicina), as duas últimas ficaram no percentil de $70 \%$ de acertos e a primeira abaixo deste valor. Isso pode significar, também, deficiência da intervenção educativa.

Os tópicos tecido necrótico e pomada neomicina foram os que revelaram a melhor evolução em relação ao nível de conhecimento após intervenção educativa: $40,2 \%$ e $39,2 \%$ de acertos a mais do que os encontrados no pré-teste, indicando melhoria expressiva do nível de conhecimento. Ainda podem-se destacar os tópicos 1, 7, 12 e 13, os quais marcaram um aumento de acertos de 31\%, 35\%, 30,4\% e 30,4\% respectivamente. Esses valores corroboram importante aumento da compreensão a respeito dos métodos para a limpeza de feridas, resposta ao tratamento instituído, uso de petrolatos e AGE no tratamento das feridas.

Contudo, uma explicação racional para esses achados não é clara. Infere-se que determinados assuntos sejam de maior interesse dos profissionais; que eles representem a prática dos sujeitos ou que a forma de apresentá-los aos participantes não seja a mais adequada. Em que pese essas especulações, estudos com outros desenhos são requeridos para possibilitarem esclarecimentos desses pontos.

A avaliação da evolução da ferida, tópico 6, destacou-se por ser a que atingiu o maior percentual de acertos no pré-teste, evidenciando um bom conhecimento prévio dos participantes (87\%) sobre o tópico. Percebe-se que no pós-teste - embora com 100\% de acertos -, este tópico não alcançou p-valor estatisticamente significante. Isto se deve à reduzida diferença entre o conhecimento antes e após a 
intervenção educativa: 13\% apenas. Questões com altos índices de acertos, tanto no pré quanto no pós-treinamento, sugerem quatro possíveis explicações: primeiro, que os sujeitos já possuíam informações sobre o tema (mais provável para o caso), aspecto positivo, pois o reforço das informações conhecidas ajuda a fixar o conteúdo e relacioná-lo a novas informações; segundo, houve erro na avaliação de necessidade da intervenção educativa; terceiro, que os participantes não constituíam o público-alvo correto; quarto, que os instrutores não souberam elaborar o conteúdo na medida da necessidade dos profissionais ${ }^{2}$.

O levantamento e a avaliação de necessidades de treinamentos são os primeiros e uns dos mais determinantes passos do planejamento de uma atividade educativa, que envolvem a disponibilidade e o preparo dos profissionais para reconhecerem suas limitações e necessidades de capacitação. Esta avaliação está associada, em parte, à integração das equipes com o pessoal dos serviços de educação continuada. Outra forma adotada para avaliação de necessidades de treinamentos é a análise dos indicadores de qualidade institucionais, como índices de infecções, flebites e úlcera por pressão ${ }^{2}$.

$O$ processo de avaliação de treinamento e desenvolvimento pode ser realizado em quatro níveis de avaliação: reação ou satisfação (opinião do participante acerca das condições para aprendizagem); aprendizagem (eficácia do treinamento no que se refere à aquisição ou aumento do conhecimento); comportamento (mudanças geradas pelo treinamento no comportamento dos participantes); e resultados (transformação na prática do trabalho) ${ }^{12}$. Neste estudo, adotou-se a avaliação da aprendizagem, dada a dificuldade de se alcançar os dois últimos no cuidado ao paciente com feridas em atenção primária na realidade estudada.

Diante da necessidade de identificação e avaliação de novos métodos que favoreçam a aquisição de conhecimentos e uma possível mudança na prática da equipe técnica de enfermagem frente às questões relacionadas ao cuidado de enfermagem com paciente com feridas, é importante investigar o impacto dessas estratégias de educação nas atitudes dos profissionais.

Esta pesquisa contém algumas limitações: a amostra foi pequena, não foi avaliado se a aquisição de conhecimento incrementou mudanças na prática. estudo precisa ser replicado em caráter multicêntrico, com amostra representativa, incluindo todos os membros da equipe de enfermagem, em que pese as adaptações do instrumento às populações e realidades investigadas.

Apesar desses apontamentos que limitam a generalização dos resultados, este estudo traz avanços para a enfermagem em diversos campos. Na gestão, esta foi a primeira investigação que avaliou e demonstrou o impacto positivo da educação em saúde no conhecimento sobre feridas dos técnicos de enfermagem de atenção primária, fortalecendo as políticas de educação permanente, tão importantes e igualmente escassas nas instituições de saúde para o cuidado aos portadores de feridas. A pesquisa subsidiou o conhecimento e apontou caminhos para novas investigações na área, a exemplo da avaliação do impacto da intervenção na prática em situação real de assistência. No ensino, apontou os temas de maior dificuldade para compreensão e a necessidade da implementação de estratégias coadjuvantes que potencializem o aprendizado. Na prática, como conhecimento ideal é parte essencial de práticas adequadas, infere-se que os participantes ampliaram as aptidões para assistência holística.

\section{Conclusão}

Conclui-se que a intervenção educativa proporcionou melhora no nível de conhecimento dos estudados sobre avaliação, manejo e tratamento tópico de feridas. Entretanto, é preciso buscar estratégias coadjuvantes, inovadoras ou não, a fim de proporcionar maior nível de conhecimento. Entre os temas que requerem maior atenção das estratégias de ensino-aprendizagem estão o desbridamento autolítico, ferida com cavidade e tecido com granulação, tópicos com menor percentual de aprendizagem neste estudo. Pesquisas de natureza qualitativa podem ser oportunas para se compreender as dificuldades para aprender, assimilar ou aceitar as recomendações advindas desses tópicos.

\section{REFERÊNCIAS}

1.Jacondino CB, Severo DF, Rodrigues KR, Lima L, Einhardt RR, Amestoy SC. Educação em serviço: qualificação da equipe de enfermagem para o tratamento de feridas. Cogitare Enferm. 2010; 15:314-8.

2.Mira VL, Follador NN, Ferrari CRS, Oliveira LFMN, Silva JAM, Santos PT. Evaluation of effectiveness of training of nursing professionals: a correlational study. Online Braz J Nurs. 2012; 11:595-606.

3.Oliveira BGRB, Castro JBA, Granjeiro JM. Panorama epidemiológico e clínico de pacientes com feridas crônicas tratados em ambulatório. Rev enferm UERJ. 2013; 2:612-7.

4.Ferreira AM, Rigotti MA, Pena SB, Paula DS, Ramos IB, Sasaki VDM. Conhecimento e prática de acadêmicos de enfermagem sobre cuidados com portadores de feridas. Esc Anna Nery. 2013; 17:211-9.

5.Ashton J, Price P. Survey comparing clinicians' wound healing knowledge and practice. Br J Nurs. 2006; 15(19):S18-S26.

6.Sinclair L, Berwiczonek H, Thurston N, Butler S, Bulloch G, Ellery C, et al. Evaluation of an evidencebased education program for pressure ulcer prevention. J Wound, Ostomy, and Continence Nurs. 2004; 31:43-50. 7.Fernandes LM, Caliri MHL, Haas VJ. The effect of 
educative interventions on the pressure ulcer prevention knowledge of nursing professionals. Acta Paul Enferm. 2008; 21:305-11.

8.Zulkowski K, Ayello EA, Wexler S. Certification and education: do they affect pressure ulcer knowledge in nursing? J Nurs Adm. 2010; 40(10 Suppl): S28-32.

9.Bastos LF, Ciampone MH, Mira VL. Asessment of evaluation of transference support and training impact on the work of nurses. Rev Latino-Am Enfermagem. 2013; 21:1274-81.

10.Ministério da Saúde (Br). Portaria n ${ }^{\circ}$ 1.996/GM, de 20 de agosto de 2007. Dispõe sobre as diretrizes para a implementação da Política Nacional de Educação Permanente em Saúde e dá outras providências. Brasília(DF): Gabinete Ministerial; 2007.

11.Ministério da Saúde (Br). Portaria nº 198/GM, de 13 de fevereiro de 2004. Institui a Política Nacional de Educação Permanente em Saúde como estratégia do Sistema Único de Saúde para a formação e o desenvolvimento de trabalhadores para o setor e dá outras providências. Brasília(DF); Gabinete Ministerial; 2004.

12.Mira VL, Peduzzi M, Melleiro MM, Tronchin DMR, Prado MFF, Santos PT et al. Analysis of the learning evaluation process of nursing staff actions. Rev esc enferm USP. 2011; 45:1574-81.

13.Meneses PPM, Zerbini T. Levantamento de necessidades de treinamento: reflexões atuais. Análise. 2009; 20(2):50-64.

14. Tamayo N, Abbad GS. Autoconceito profissional e suporte à transferência e impacto do treinamento no trabalho. Rev Adm Contemp. 2006; 10(3):9-28.

15.Santos AAR, Medeiros ABA, Soares MJGO, Costa MML. Avaliação e tratamento de feridas: o conhecimento de acadêmicos de enfermagem. Rev enferm UERJ. 2010; 18:547-52.

16.Miyazaki MY, Caliri MH, dos Santos CB. Knowledge on pressure ulcer prevention among nursing professionals. Rev Lat-Am Enfermagem. 2010; 18:1203-11. 